\title{
Una exploración de las adaptaciones culturales prehistóricas en Baja California
}

\author{
Don Laylander \\ Centro de Administración de Recursos Culturales \\ Unversidad Estatal de San Diego
}

\section{Resumen}

Esta investigación profundiza acerca de cuáles cambios y cuándo ocurrieron en los estilos y las tecnologías prehistóricas tal como se ven reflejadas en la cultura material que se ha conservado. Al mismo tiempo analiza cómo fue que las sociedades lograron establecerse aquí y cómo fue que pudieron sostenerse y desarrollarse en este medio ecológico, o en su caso, por qué fracasaron en su intento. Con el fin de obtener información acerca de las adaptaciones culturales prehistóricas se recurre a la evidencia etnográfica y lingüística, y a la evidencia arqueológica. Otra fuente de información de las adaptaciones culturales de la Baja California es la analogía etnográfica general. Finalmente se hace un resumen de lo que fueron estas adaptaciones a modo de propuesta, con el fin de que la información resultante sea de utilidad para antropólogos y arqueólogos que partiendo del escrutinio de ésta contribuyan al avance del conocimiento de la prehistoria de esta región.

Palabras clave: exploración, adaptaciones culturales prehistóricas, evidencias arqueológicas, etnografía, lingüística.

\begin{abstract}
This research deepens on the changes happened to the styles and prehistoric technologies, and when did they take place, to get to the form they are reflected in the material culture that is still conserved. At the same time, it analyzes the reasons why these societies established in this region, how were they able to survive and to develop themselves in this ecological environment; or on the contrary, why did they failed on their trial. In order to obtain information regarding prehistoric cultural adaptations, this essay uses ethnographic and linguistic as well as archeological evidences. Other source of information of cultural adaptations in Baja California is the general ethnographic analogy. It concludes with a summary and a proposal. Anthropologists and archeologists would find useful the information herein obtained, who if they continue researching on this proposal may contribute to the continuous knowledge of the prehistory of this region.
\end{abstract}

Keywords: exploration, prehistoric cultural adaptations, archeological evidences, ethnography, linguistics. 


\title{
UNA EXPLORACION DE LAS ADAPTACIONES CULTURALES PREHISTORICAS EN BAJA CALIFORNIA*
}

\author{
Por \\ Don Laylander**
}

\section{INTRODUCCION}

Antes que nada, es necesario considerar las implicaciones que tiene el título de este trabajo. Geográficamente, se refiere al estado de Baja California; en términos de la cronología, comprende alrededor del $98 \%$ de la historia del mismo antes de que las fuertes influencias culturales europeas y del centro de México empezaran a sentirse.

En su artículo "Algunas observaciones sobre los complejos arqueológicos de Baja California" (Bendímez, 1985), la arqueóloga Julia Bendímez hábilmente resumió la secuencia cronológica de los complejos arqueológicos de la región como se conocen hoy en día. Tal cronología cultural comprende las nociones que algunos investigadores tienen acerca de la arqueología de este territorio, y es al menos el punto de partida para entender la prehistoria, pero no la única perspectiva. Es importante saber qué cambios ocurrieron en los estilos y las tecnologías prehistóricas tal y como son reflejadas en la cultura material que se ha conservado, así como cuándo se llevaron a cabo esos cambios. También es importante conocer por qué se dieron esos cambios y por qué no se dieron otros; en otras palabras, queremos analizar cómo fue que las sociedades lograron establecerse aquí y cómo fue que pudieron sostenerse y desarrollarse en este medio ecológico, o en su caso, por qué fracasaron en su intento. Esto aconteció a través de un sistema de ideas compartidas denominado cultura, $\mathrm{y}$ a través de un proceso mediante el cual los elementos de la cultura se adecuaron al entorno físico y social denominados adaptación cultural.

Al intentar obtener información acerca de las adaptacionesculturales prehistóricas, no nos limitamos a la evidencia arqueológica, también recurrimos a la evidencia etnográfica y lingüística (Laylander, 1987). Al decir que la etnografía y la lingüística son relevantes en el estudio de la prehistoria, desde luego no estamos sugiriendo que los pueblos con los que han trabajado los antropólogos durante épocas recientes, tanto mexicanos como extranjeros, sean de carácter "prehistórico"; así como tampoco lo son los pueblos descritos por los exploradores y los misioneros jesuitas, franciscanos y dominicos. Sin embargo, indudablemente existieron continuidades culturales importantes entre los pueblos aborígenes conocidos históricamente en Baja California y sus ancestros prehis-

\footnotetext{
* Traducido del inglés por Héctor León y Julia Bendimez

** Antropólogo, actualmente presta servicios en el Centro de Administración de Recursos Culturales de la Universidad Estatal de San Diego, California.
} 
tóricos. Si se pudieran diferenciar los cambios que ocurrieron en el período histórico - cambios que fueron abundantes, importantes y algunas veces engañosos - entonces la antropología del período histórico, junto con la arqueología, pudieran ser uno de los pilares más importantes para la reconstrucción del pasado prehistórico.

Otra fuente que provee información acerca de las adaptaciones culturales prehistóricas en Baja California es la analogía etnográfica general. Con esto nos referimos al hecho de que a nivel mundial, se conoce bastante acerca de cómo otras sociedades con tecnologías similares se adaptaron culturalmente a medios ecológicos parecidos. Con algo de precaución se pueden tomaren consideración tales ejemplos y patrones al intentar completar una visión de la Baja California prehistórica.

Nuestro último comentario explicatorio atañe al énfasis en el título "exploración" acerca de las adaptaciones culturales prehistóricas. Intentaremos hacer un resumen de lo que fueron esas adaptaciones, admitiendo que lo consideramos aún sólo como una propuesta. Sin embargo, creemos que esta información será de utilidad para antropólogos y arqueólogos que parten del escrutinio de ésta y de la que se adquiera en los próximos años, para contribuir al avance del conocimiento de la prehistoria de esta región.

\section{PERIODIZACION Y REGIONALIZACION}

Se puede considerar la prehistoria de Baja California principalmente en tres períodos: paleoindígena, arcaico y prehistórico tardío. También se pueden distinguir tres subregiones geográficas: el noroeste, el noreste y el sur. En este contexto, no es necesario considerar el tema de las adaptaciones culturales del llamado período "temprano" de la prehistoria. A algunos sitios se les ha atribuido una gran antigüedad; entre éstos se pueden mencionar los sitios de Cálico, la isla de Santa Rosa y Yuha, al norte de la frontera con Baja California, así como la laguna Seca de Chapala en el estado. El problema con todas las culturas de más de 12,000 años de antigüedad en esta parte del mundo, es que se tiene que probar si realmente existieron o no. Las especulaciones acerca de los detalles de adaptación parecen por ahora prematuras.'

Del período paleoindígena se cuenta con escasa evidencia arqueológica. En el centro de la península de Baja California se ha reportado el hallazgo de una punta de lanza o de dardo que muestra las características del estilo Clovis. Otras han sido reportadas en el sur de California, Nevada y Arizona, Estados Unidos (Aschmann, 1952, 17: 262-263; Davis y Shutler, 1969, 14: 154-169). Con base en éstas, se puede proponer la existencia de una temprana y breve ocupación de Baja California por pueblos que estaban culturalmente emparentados con los cazadores de la megafauna del centro de los Estados Unidos y del sur de México. Es viable la teoría de que estos antiguos cazadores de la cultura Clovis

\footnotetext{
I Sin embargo, se han propuesto tales especulaciones, como por ejemplo: Davis, 1978.
} 
hayan estado organizados en pequeños grupos, sin arraigo firme en una localidad en particular o en una extensión territorial limitada, sino que se mantenían en continuo movimiento, siguiendo los vestigios de la megafauna del pleistoceno dondequiera que la oportunidad se presentara. Eléxito de esta adaptación tal vez fue a corto plazo, aunque pudo haber sido demasiado superficial para mantener un control del entorno y por otro lado, podría haber causado su destitución por el abuso de la cacería. Por estas razones, posiblemente no se presentó una continuidad con las culturas posteriores, pero esto resulta ser una mera especulación con base en una sola punta del estilo Clovis.

El otro complejo paleoindígena, algunas veces llamado San Dieguito o lago Mohave, ofrece más evidencia, aunque también es altamente especulativa (por ejemplo: Rogers, 1966; Warren, 1967, 32: 168-185; Warren, 1966; Kaldenber, 1982). Sitios arqueológicos reportados como pertenecientes a estecomplejo son más numerosos en el noroeste de la península, aunque también se les encuentra en el noreste y posiblemente en porciones del sur de ésta.

Desafortunadamente existe todavía una considerable incertidumbre acerca de la naturaleza de estos sitios del complejo San Dieguito. Por ejemplo, si representan campamentos temporales de pequeños grupos de cazadores, como los propuestos para la cultura Clovis, o si éstos tuvieron un objetivo en especial para comunidades más numerosas. Tal vez lo más que se puede hacer por ahora, al establecer conjeturas acerca de las adaptaciones de los habitantes de San Dieguito, es proponer que estos pueblos también vivían en pequeños grupos pero más estacionarios que los grupos de Clovis, que seguían cazando animales grandes pero también incluían una gran variedad de animales pequeños, plantas y recursos marinos. Con esto, los bajacalifomianos prehistóricos pertenecientes al período paleoindigena empezaban a diversificar sus habilidades y a explotar en forma más extensa los recursos del medio ambiente. A este respecto, se da un sentido de continuidad en el desarrollo de estos pueblos y de sus aún más versátiles y localmente adaptados sucesores.

En la arqueología, las necesidades más apremiantes para el período paleoindígena son las de separar y definir mejor las características culturales. De esta manera, se ayudaría a identificar el complejo San Dieguito y a encontrar más sitios, que con mayor precisión pudieran ser fechados como pertenecientes a ese período. Es muy posible que al menos algunos de los sitios que anteriormente eran clasificados como pertenecientes al complejo San Dieguito realmente corresponden a un periodo posterior. También con excavaciones mejor documentadas y mediante análisis de los objetos recolectados en los trabajos de excavación y de reconocimiento de superficie, debe ser posible definir más claramente el campo de actividades en localidades específicas, así como la manera en que las comunidades enteras se ajustaban al entorno, que cambiabc durante ese período.

Para el período arcaico, que comprende un lapso desde hace 8,000 año: hasta hace 1,500 aproximadamente, la evidencia de los complejos La Jolla j Amargosa es más extensa, aunque su interpretación sigue siendo difícil (po] 
ejemplo: Moratto, 1984; Chartkoff y Chartkoff, 1984; Massey, 1966, 4: 38-58). Se puede sugerir que lingüísticamente, los antepasados de los grupos históricos yumano y cochimí ya estaban asentados en sus lugares en este período (Laylander, 1985: 14-58). Algunos de los rasgos culturales característicos de estos pueblos sin duda ya estaban presentesen tiempos arcaicos, aunque ha sido poca la información inferida de los registros etnográficos de una forma sistemática.

Los grupos arcaicos son mejor conocidos arqueológicamente, como los que formaron los depósitos de concha de molusco que se encuentran a lo largo de las costas de Baja California. En tal sentido, la península es mejor conocida por su espectacular arte rupestre, pero se puede sugerir que estas áreas de desecho de conchas, aunque menos espectaculares $-\mathrm{y}$ desafortunadamente tan frágiles como las primeras-, podrian ayudar a conocer la prehistoria y quizá proporcionarían más información que las pinturas rupestres. A partirde estas acumulaciones de desechode moluscos, el arqueólogo podría obtenerinformación que aclarara datos como las áreas que fueron ocupadas y cuándo, las estaciones del año en que los moluscos fueron recolectados (Killingley, 1980: 19-23), las temperaturas de la costa y las características físicas del entorno en términos de promontorios rocosos, playas o lagunas. En circunstancias favorables, los montones de concha pueden dar algunas bases sobre las cuales se podría calcular el tamaño de los grupos sociales que recolectaban y la manera en que eran transportados o intercambiados estos moluscos con grupos del interior de la península, obteniendo a cambio, por ejemplo, materia prima para la elaboración de herramientas. Los entierros que algunas veces son encontrados en estos depósitos, pueden dar información no sólo acerca de las características raciales, salud y longevidad, sino también las posibles diferencias de status entre los sexos y de edad entre los individuos, así como acerca del poder de los sistemas rituales que son reflejados en la postura, orientación y artículos funerarios.

Lo que ya se conoce sobre el período arcaico de Baja California da una idea de que en la mayoría de los diferentes medios ecológicos - quizá en todos pero principalmente a lo largo de las costas - las poblaciones que eran de un tamaño mediano utilizaban una extensa variedad de recursos alimenticios, particularmente plantas y moluscos. Existían sistemas de trueque, aunque parece ser que éstos no eran muy frecuentes; cada comunidad era en gran medida autosuficiente. La tecnología, manifestada por los rasgos que han sobrevivido, parece haber sido bastante simple y conservadora.

El período de la prehistoria tardía, que inicia hace 1,500 años cuando menos, es particularmente interesante, tanto por sus características como por su enorme evidencia disponible para ser estudiada. El sur fue la región del complejo Comondú (Massey, 1978) y del idioma cochimí, y es en esta región en donde encontramos una menor variación entre las sociedades desde el período arcaico hasta la prehistoria tardía. En este caso, la adaptación parece haber continuado fundamentalmente en pequeños grupos generalizados y autosuficientes. Sin embargo, es también la subregión (y probablemente éste fue el período) en que floreció la pintura rupestre representativa (Meighan, 1969; Grant, 1974; Hambleton, 1979; Crosby, 1984).¿Qué pudo haber estimulado este 
florecimiento artístico? ¿fue una respuesta desesperada a alguna crisis de adaptación a la cual los cochimíes tuvieron que enfrentarse en relación con un medio ambiente en deterioro, o en el equilibrio político entre grupos diferentes en competencia entre sí? Cuando los misioneros llegaron, este movimiento artístico parece ser que ya era cosa del pasado, así que la única manera de comprenderlo es a través de la arqueología. Esto tendría que involucrartanto un análisis completo y riguroso del arte rupestre, como la investigación de los vestigios de los asentamientos cochimíes asociados a ellos.

En el noroeste y noreste, de acuerdo con los patrones de la prehistoria de Baja California, el período de la prehistoria tardía fue uno de los que mostró cambios más revolucionarios (Rogers, 1966: 167-198). En algunas regiones, en la parte tardía del período arcaico aparecieron el mortero y el procesamiento de la bellota. Donde quiera que se le pudiera encontrar, la bellota ofrecía una fuente alimenticia relativamente abundante, nutritiva y fácil de almacenary, además, adecuada para cubrir las necesidades durante las épocas de escasez de alimentos. Esto a su vez habría fomentado o hecho posible un mayor número de asentamientos (y más permanentes), una diferenciación en los roles o estratos sociales, y una propiedad más exclusiva del territorio. Otras innovaciones fueron el arco y la flecha, que hicieron posible una mayor eficiencia en la cacería y en la guerra. La cerámica, por otro lado, posibilitó la conservación y el transporte de comestibles y de agua. Todos estos cambios promovieron el sedentarismo y, a su vez, el sedentarismo provocó cambios en los primeros. En el noreste del estado, la introducción de la agricultura dio lugar a otro cambio revolucionario en la adaptación y promovió aún más la sedentarización de poblaciones más densas, la exclusividad territorial y la diferenciación social. El panorama que tenemos de las adaptaciones culturales en el noroeste y en el noreste durante el período de la prehistoria tardía, es el de poblaciones en crecimiento que estaban en proceso de separación lingüística, así como en términos sociales tendian a formar grupos más pequeños y más sedentarios, capaces de explotar ciertos recursos naturales con mayor grado de intensidad, pero en consecuencia, cada vez más integrados en sistemas económico-sociales por la necesidad de conseguir acceso o para intercambiar artículos difíciles de suministrarse localmente. En el noreste los grupos estaban también ligados por la necesidad de alianzas militares para mantener el control de los terrenos agrícolas, un nuevo y escaso recurso de gran valor.

Los sitios prehistóricos del período de la prehistoria tardía son interesantes, sobre todo por las posibilidades que ofrecen para estudiar e interpretar la extensión de innovaciones tecnológicas, y por las posibilidades de reconstruir las rutas de intercambio de obsidiana, de cerámica y de moluscos, por ejemplo. Muchos de tales sitios se encuentran en casi todas las regiones del estado, pero hasta la fecha muy pocos han sido investigados. No obstante, como los sitios de épocas anteriores, su número inexorablemente ha ido decreciendo por la acción del vandalismo y por la apertura de estos terrenos a su utilización moderna.

Los estudios lingüísticos y etnográficos, sobre todo los de los grupos yumanos que habitaban en el noroeste y en el noreste, han conservado mucha 
información, misma que espera ser analizada y de la cual se pueden obtener datos acerca de los cambios en las adaptaciones prehistóricas en estas áreas (Hicks, 1963; Kelly, 1977; Ochoa Zazueta, 1978; Crawford, 1983; Mixco, 1983).

Para resumir, Baja California goza de un registro arqueológico y etnográfico muy rico, que puedeservir de modelo para medir las adaptaciones culturales. Se han hecho muchas contribuciones importantes durante las últimas décadas en cuanto a la exploración y esclarecimiento de ese registro; sin embargo, todavía no se ha dado más que un paso. Los modelos de adaptación cultural aquí señalados brevemente, son cuando más, meros intentos que hacen juego con lo que se sabe de la Baja California y las regiones aledañas, los cuales necesitan revisarse minuciosamente en un futuro próximo.

Para generalizar, una imagen que se puede proponer de la prehistoria de Baja California es la de continuidad, pero no necesariamente de estabilidad. No hay evidencia indiscutible de que haya habido alguna interrupción en las ocupaciones del estado o de sus subregiones más importantes durante la época posterior a la Clovis. Parece ser que los cambios de adaptación tuvieron su origen tanto dentro como fuera de la región.

Sin embargo, estas adaptaciones no lograron mantener el control sobre la península ante los desafíos culturales que llegaron del exterior durante la época histórica. A pesar de esto, estas tradiciones culturales prehistóricas siguen siendo uno de los elementos de la herencia cultural bajacaliforniana. También constituyen un recordatorio de lo complejo, y algunas veces frágil, que resulta ser el medio ambiente. 


\section{BIBLIOGRAFIA}

ASCHMANN, Homer. 1952. "A Fluted Point From Central Baja California", American Antiquity 17.

BENDIMEZ, Mary Julita. 1985. “Algunas observaciones sobre la arqueología de Baja California”, Cuarto Simposium de Historia Regional, Ensenada, 1985; Revista Meyibó, UABC, vol. II, № 5.

CRAWFORD, James H. 1983. Cocopa Texts, Berkeley, University of California Press.

CROSBY, Harry. 1984. The Cave Paintings of Baja California, Salt Lake Copley.

CHARTKOFF, Joseph L. y Kerry Kona Chartkoff. 1984. The Archaeology of California, Stanford.

DAVIS, E.L. y R. Shutler, Jr. 1969. "Recent Discoveries of Fluted Points in California and Nevada", Nevada State Museum Anthropological Papers 14.

GRANT, Campbell. 1974. Rock Art of Baja California, Los Angeles.

HAMBLETON, Enrique. 1979. La pintura rupestre de Baja California. Banamex. México.

HICKS, Frederic N. 1963. Ecological Aspects of Aboriginal Culture in the Western Yuma Area. Disertación doctoral, University of California, Los Angeles.

KALDENBERG, Rusell L. 1982. "Rancho Park North: A San Diego Dieguito La Jolla Shellfih Processing Site in Coastal Southern California". Imperial Valley College Museum Society Ocassional Paper, 6.

KELLY, William H. 1977. "Cocopa Ethnography”, Anthropological Papers of the University of Arizona, University of Arizona Press, 29.

KILLINGLEY, J.S. 1980. "Seasonality of Mollusk Collecting at Hubby Midden Site 1959: VI: 28A, Pacific Coast Archaeological Society Quarterly, 16.

LAYLANDER, D. 1985. "Some Lingüistic Approaches to Southern California's Prehistory", Cultural Resources Management Enter Casual Papers, 2. San Diego State University. 1987. Sources and Strategies for the Prehistory of Baja California. Tesis de maestría, San Diego State University.

DAVIS, Emma Lou. 1978. The Ancient Californians: Rancholabrean Hunters of the Majave Lakes Country, Natural History Museum of Los Angeles Country.

MASSEY, William C. 1966. "Archaeology and Ethnohistory of Lower California”. Handbook of Middle American Indians, vol. 4: 38-58, Robert Wauchupe, Ed. Austin, University of Texas Press.

MEIGHAN, Clement W. 1969. Indian Art and History: The Testimony of Prehistoric Rock Paintings in Baja California, Los Angeles, Dawson's.

MIXCO, Mauricio J. 1983. "Kiliwa Texts", University of Utah, Anthropological Papers, 107.

MORATTO, Michael J. 1984. California Archaeology, Orlando.

OCHOA Zazueta, Jesús Angel. 1978. Los kiliwa y el mundo se hizo asi, México.

RAYMOND Tuohy, Donald. Culture History in the Comondu Region, Baja California, Mexico. Tesis de maestría, University of Nevada, Las Vegas. 
ROGERS, Malcolm J. 1945. "An Outline of Yuman Prehistory". Southwestern Journal of Anthropology, 1. 1966. Ancient Hunters of the Far West, Copley, San Diego.

WARREN, Claude N. 1967. "The San Dieguito Complex: A Review and Hypothesis", American Antiquity, 32. 1966. "The San Dieguito TypeSite: M.J. Rogers 1938 Excavation on the San Dieguito River”, San Diego Museum Papers, 5. 